\title{
Simpson Grade 1
}

National Cancer Institute

\section{Source}

National Cancer Institute. Simpson Grade 1. NCI Thesaurus. Code C128617.

Macroscopically complete removal of the tumor, with excision of its dural attachment, and of any abnormal bone. Includes resection of venous sinus if involved. 